\title{
Risk of severe maternal morbidity associated with cesarean delivery and the role of maternal age: a population-based propensity score analysis
}

\author{
Diane Korb MD MPH, François Goffinet MD PhD, Aurélien Seco MSc, Sylvie Chevret MD PhD, Catherine Deneux- \\ Tharaux MD PhD; for the EPIMOMS Study Group
}

Cite as: CMAJ 2019 April 1;191:E352-60. doi: 10.1503/cmaj.181067

\begin{abstract}
BACKGROUND: Short-term maternal complications of cesarean delivery remain uncertain because of confounding by indication. Our objective was to assess whether cesarean delivery is associated with severe acute intra- or postpartum maternal morbidity compared with vaginal delivery, overall and according to the timing of the cesarean.
\end{abstract}

METHODS: We performed a case-control analysis using data from EPIMOMS, a prospective population-based study of deliveries at 22 gestation weeks or later from 6 regions of France in 2012-2013. Cases of intra- or postpartum severe acute maternal morbidity that were not a result of a condition present before delivery were compared with controls randomly selected in a $1 / 50$ ratio. Associations between delivery modes and severe acute maternal morbidity were estimated in a propensity score-matched sample.

RESULTS: Among 182300 deliveries, we identified 1444 cases and 3464 controls. The proportion of cesarean delivery was significantly higher among cases than controls (36.0\% v. $18.2 \%)$. In the propensity score-matched analysis, cesarean deliveries were significantly associated with a higher risk of severe acute maternal morbidity (adjusted odds ratio [OR] $1.8,95 \%$ confidence interval $[\mathrm{Cl}] 1.5-2.2$ ).
This association increased with maternal age and was particularly marked for women aged 35 years or older (adjusted OR 2.9, 95\% Cl 1.9-4.4). This increased risk was significant for cesarean deliveries during labour in women of all age groups and for those before labour only in women aged 35 years or older (adjusted OR 5.1, 95\% Cl 2.3-11.0).

INTERPRETATION: Cesarean delivery is associated with a higher risk of severe acute maternal morbidity than vaginal delivery, particularly in women aged 35 years and older. Clinical decisions regarding delivery mode should account for this excess risk accordingly. esarean delivery is a useful intervention for mothers and newborns in many situations. Nonetheless, its rates have soared over the past 20 years in most developed countries, where more than 1 out of 5 women deliver by cesarean. ${ }^{1}$ The range of indications for cesarean delivery appears to have broadened considerably, with more cesarean deliveries likely to be performed for questionable medical indications..$^{2-7}$ This increase requires evaluation of its potential adverse consequences on maternal and neonatal health.

The long-term obstetric risks associated with the presence of a scarred uterus in future pregnancies are well recognized, primarily uterine rupture and abnormal placentation. ${ }^{8-13}$ Conversely, conclusions about the comparative short-term maternal risks of cesarean and vaginal delivery remain unclear. A randomized controlled trial among women with no medical indication for cesarean delivery is, at best, ethically questionable. Observational studies can provide relevant information to address this, but their conclusions are likely to be limited owing to confounding by indication. That is, the fact that maternal morbidity may be a result of the condition indicating or justifying the cesarean delivery rather than to the surgical procedure itself can produce an apparent association between cesarean delivery and maternal morbidity.

Earlier studies of the association between maternal mortality and mode of delivery have shown a higher risk of maternal mortality associated with cesarean versus vaginal deliveries. ${ }^{14,15}$ These studies, however, were limited by their retrospective design, the rarity of maternal deaths and insufficient consideration of this confounding by indication. Over the past 10 years, numerous observational studies have reported discordant results about the association between cesarean and severe maternal morbidity, and their conclusions too are limited by several methodological flaws: insufficient control for confounding by indication, inappropriate definition of severe acute maternal morbidity, retrospective designs limiting quality and availability 
of data, non-population-based designs restricting the generalizability of results and failure to differentiate between cesarean delivery before or during labour. ${ }^{16-20}$

One of the aims of the EPIMOMS prospective populationbased study, which was specifically designed to study severe acute maternal morbidity, was to explore its association with cesarean delivery. Our objective here is to test for - and if it exists, quantify - the association between intra- or postpartum severe acute maternal morbidity and cesarean versus vaginal delivery, overall and according to the timing of cesarean delivery, before or during labour. This analysis focused especially on controlling confounding by indication, first by careful selection of the women analyzed, excluding situations at high risk of confounding by indication, and then by using propensity scores to control for residual confounding by indication.

\section{Methods}

\section{Study population}

We designed an unmatched case-control analysis within the EPIMOMS study, a prospective population-based study conducted in 6 French regions from May 1, 2012, through Nov. 30, 2013. Recruitment took place over a 1-year period in each region at 119 maternity units and 136 intensive care units (ICUs) that accounted for 182309 deliveries during the study period - i.e., one-fifth of those in France during the study period - with characteristics of parturients, hospitals and rates of cesarean deliveries similar to the national profile. ${ }^{21}$ The EPIMOMS study includes a standardized definition of severe acute maternal morbidity that was developed through a national Delphi process to gain formal expert consensus, intended to characterize maternal complications with severe health alteration and organ dysfunction. The EPIMOMS multicriteria definition combines diagnoses (severe obstetric hemorrhage, eclampsia, severe preeclampsia, pulmonary embolism, stroke and psychiatric disorder), organ dysfunctions (hepatic, hematological, respiratory, cardiovascular, renal and neurologic), and interventions (admission to ICU and laparotomy after delivery) (definition detailed in Appendix 1, available at www.cmaj.ca/lookup/suppl/doi:10.1503/ cmaj.181067/-/DC1).

\section{Definition of cases and controls}

We prospectively identified all women who had a severe acute maternal morbidity event during pregnancy (after 22 weeks of gestation) or in the 42 days postpartum and included them in the EPIMOMS study $(n=2540)$. We controlled the completeness of case identification through the review of delivery logbooks, hospital discharge data and laboratory files; we included additional cases after their eligibility was confirmed by the clinicians in charge.

For the present analysis, we excluded women with antepartum severe acute maternal morbidity and defined other exclusion criteria to focus on the population for which the alternate between cesarean and vaginal delivery exists, so women in situations in which 1 mode of delivery is systematically used were excluded. Other exclusion criteria included obstetric conditions that developed during pregnancy that were symptomatic and present before labour and responsible for postpartum severe acute maternal morbidity (e.g., placenta previa responsible for a severe postpartum hemorrhage, or preeclampsia diagnosed during pregnancy and meeting severe acute maternal morbidity criteria in the postpartum); and severe acute maternal morbidity during labour that required an emergency cesarean. The remaining women with intrapartum or postpartum severe acute maternal morbidity were the cases used for our analysis.

Concomitantly, as per the EPIMOMS study protocol, from the hospitals' delivery files we selected a $1 / 50$ random sample of women who delivered without severe acute maternal morbidity in the same regions during the same time period as the control group. We determined this control sample size to be able to show a relative risk of 1.5 or more for a characteristic present in $10 \%$ or more of the women with $\alpha=0.05$ and $1-\beta=0.9$; with an expected incidence of severe acute maternal morbidity of $1 \%$ of deliveries, the inclusion of $2 \%$ of deliveries without severe acute maternal morbidity - i.e., 1/50 - in the control group was needed. Similar exclusion criteria as for the cases were applied.

\section{Definition and measurement of exposures}

For all women with severe acute maternal morbidity and those of the representative control sample included in the EPIMOMS study, we collected data on the women's social and demographic characteristics, pre-existing medical and obstetric conditions, characteristics and complications of the current pregnancy and delivery, and details about the course and management of the morbidity event from medical records in which these items were prospectively recorded as they occurred. We collected data about characteristics of the maternity units in a specific questionnaire.

Mode of delivery, the exposure of interest, was studied in 2 ways. We first distinguished vaginal from cesarean deliveries. Then, to take into account the timing of the cesarean delivery, we created 3 groups: vaginal deliveries, cesarean deliveries before labour and cesarean deliveries during labour.

\section{Statistical analysis}

We compared the characteristics of cases and controls, based on $\chi^{2}$ or Fisher exact tests for categorical variables and Student $t$ or Wilcoxon rank sum tests for continuous variables, as appropriate.

To control for confounding factors that might influence both the choice of the mode of delivery and the occurrence of intra- or postpartum morbidity, we used a propensity score approach. A woman's propensity score was defined as her probability of a cesarean delivery based on her individual covariates measured before delivery. Details on the propensity score construction and matching are provided in Appendix 2 (available at www.cmaj.ca/ lookup/suppl/doi:10.1503/cmaj.181067/-/DC1). In the matched set, we used paired conditional generalized estimating equation logistic regression to estimate odds ratios (ORs) and their 95\% confidence intervals $(\mathrm{Cls})$ to quantify the association between the mode of delivery and severe acute maternal morbidity. We also performed a sensitivity analysis by inverse propensity score weighting (details of the method used are provided in Appendix 3, available at www.cmaj.ca/lookup/suppl/doi:10.1503/ cmaj.181067/-/DC1). 
We tested for clinically relevant interactions by using interaction terms between modes of delivery and the covariates considered. Because a significant positive interaction was found with maternal age, we reran the analysis after stratifying by maternal age: $<25$ years, $25-29$ years, $30-34$ years and $\geq 35$ years.

Similar propensity score analyses were performed when considering 3 modes of delivery: vaginal, cesarean delivery before labour and cesarean delivery during labour, with vaginal as the reference category, as explained in Appendix 4 (available at www.cmaj.ca/lookup/suppl/doi:10.1503/cmaj.181067/-/DC1). ${ }^{22}$

\section{Sensitivity analyses}

For testing the association between the modes of delivery (2or 3-groups exposure) and severe acute maternal morbidity, we also used a multivariable logistic regression model, based on ORs with their $95 \% \mathrm{Cls}$, adjusting for prognostic covariates. We used multilevel modelling to take into account the hierarchical structure of the data (women within maternity units) and the nonindependence of observations within maternity units.
Second, the association between the mode of delivery and severe acute maternal morbidity was tested according to the underlying causal condition by differentiating morbidity owing to severe obstetric hemorrhage and morbidity owing to other conditions by multilevel multivariable logistic modelling.

Finally, we tested the association between the planned (as opposed to the actual mode in our main analysis) mode of delivery (planned cesarean v. planned vaginal) and severe acute maternal morbidity by multilevel multivariable logistic modelling. The proportion of women with missing confounders ranged from $0 \%$ to $9.3 \%$, including 3761 (72\%) women with full data, whose characteristics were close to those of the women with missing data (data not shown). We used multiple imputation-chained equations to impute missing data. We performed all analyses with imputed data and with nonimputed data (data not shown).

All tests were 2-sided, with $p$ values of 0.05 or less denoting statistical significance. We used STATA 13 software (StataCorp LP) to conduct the descriptive and multivariate analyses, and its "psmatch2" package for matching on propensity score. ${ }^{23,24} \mathrm{We}$ used the R "Twang" and "survey" packages for the analysis with

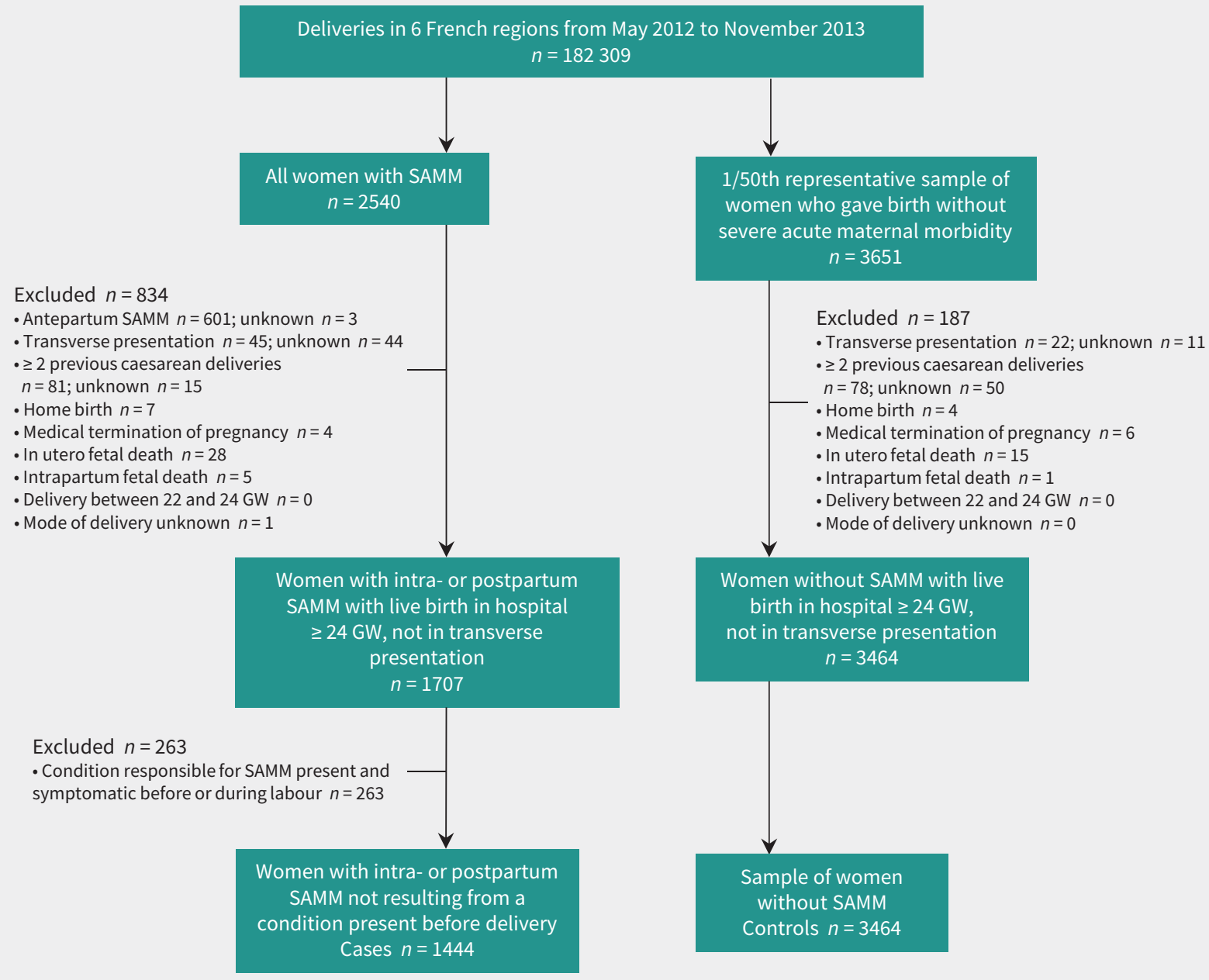

Figure 1: Flow chart showing selection of cases and controls. Note: GW = gestational week, SAMM = severe acute maternal morbidity. 
Table 1 (part 1 of 2): Characteristics of cases (women with

intra- or postpartum severe acute maternal morbidity) and controls (women who delivered without severe acute maternal morbidity)

\begin{tabular}{|c|c|c|c|}
\hline Characteristics & $\begin{array}{c}\text { No. }(\%) \text { of } \\
\text { cases }^{\star} \\
n=1444\end{array}$ & $\begin{array}{c}\text { No. }(\%) \text { of } \\
\text { controls* } \\
n=3464\end{array}$ & $\begin{array}{c}p \\
\text { value }\end{array}$ \\
\hline \multicolumn{4}{|l|}{ Maternal } \\
\hline Region or country of birth & & & 0.03 \\
\hline France & $1061(80.1)$ & $2596(83.1)$ & \\
\hline Other European country & $34(2.6)$ & $83(2.7)$ & \\
\hline North Africa & $91(6.9)$ & $209(6.7)$ & \\
\hline Sub-Saharan Africa & $79(6.0)$ & $126(4.0)$ & \\
\hline Other & $60(4.5)$ & $110(3.5)$ & \\
\hline Living without a partner & $78(5.8)$ & $128(4.0)$ & 0.01 \\
\hline \multicolumn{4}{|l|}{ Maternal age, yr } \\
\hline Mean \pm SD & $30.8 \pm 5.4$ & $30.4 \pm 5.2$ & 0.03 \\
\hline$<25$ & $211(14.7)$ & $550(15.9)$ & 0.26 \\
\hline $25-29$ & $447(31.0)$ & $1100(31.8)$ & \\
\hline $30-34$ & $467(32.3)$ & $1149(33.2)$ & \\
\hline$\geq 35$ & $319(22.1)$ & $665(19.2)$ & \\
\hline \multicolumn{4}{|l|}{ Body mass index, $\mathrm{kg} / \mathrm{m}^{2}$} \\
\hline Mean \pm SD & $24 \pm 5.1$ & $23.8 \pm 4.9$ & 0.21 \\
\hline$<18.5$ & $111(8.0)$ & $250(7.5)$ & 0.24 \\
\hline $18.5-24.9$ & $824(59.8)$ & $2050(61.2)$ & \\
\hline $25-29.9$ & $268(19.4)$ & $683(20.4)$ & \\
\hline$\geq 30$ & $176(12.8)$ & $365(10.9)$ & \\
\hline Smoker & $251(18.1)$ & $740(21.7)$ & $<0.01$ \\
\hline Pre-existing medical condition $\uparrow$ & $159(11.0)$ & $245(7.1)$ & $<0.01$ \\
\hline Parity and previous cesarean & & & $<0.01$ \\
\hline Nulliparous & $761(52.7)$ & $1495(43.2)$ & \\
\hline $\begin{array}{l}\text { Parous without previous } \\
\text { cesarean }\end{array}$ & $479(33.2)$ & $1617(46.7)$ & \\
\hline Parous with previous cesarean & $204(14.1)$ & $352(10.2)$ & \\
\hline Previous obstetric hemorrhage & $78(5.4)$ & $78(2.3)$ & $<0.01$ \\
\hline $\begin{array}{l}\text { Previous gestational } \\
\text { hypertensive disorders }\end{array}$ & $37(2.6)$ & $80(2.3)$ & 0.60 \\
\hline \multicolumn{4}{|l|}{ Pregnancy and delivery } \\
\hline In vitro fertilization & $88(6.1)$ & $73(2.1)$ & $<0.01$ \\
\hline Multiple pregnancy & $126(8.7)$ & $52(1.5)$ & $<0.01$ \\
\hline Third-trimester anemia & $329(24.4)$ & $509(16.0)$ & $<0.01$ \\
\hline Gestational hypertensive disorder & $182(12.6)$ & $215(6.2)$ & $<0.01$ \\
\hline Breech presentation & $97(6.7)$ & $140(4.0)$ & $<0.01$ \\
\hline Gestational age at delivery, GW & & & $<0.01$ \\
\hline$<32$ & $20(1.9)$ & $27(0.8)$ & \\
\hline $32-33$ & $16(1.1)$ & $24(0.7)$ & \\
\hline $34-36$ & $100(6.9)$ & $165(4.8)$ & \\
\hline$\geq 37$ & $1306(90.5)$ & $3247(93.7)$ & \\
\hline No prophylactic oxytocin after birth & $114(8.1)$ & $219(6.4)$ & 0.04 \\
\hline
\end{tabular}

Table 1 (part 2 of 2): Characteristics of cases (women with intra- or postpartum severe acute maternal morbidity) and controls (women who delivered without severe acute maternal morbidity)

\begin{tabular}{|c|c|c|c|}
\hline Characteristics & $\begin{array}{c}\text { No. }(\%) \text { of } \\
\text { cases }^{\star} \\
n=1444\end{array}$ & $\begin{array}{c}\text { No. }(\%) \text { of } \\
\text { controls* } \\
n=3464\end{array}$ & $\begin{array}{c}p \\
\text { value }\end{array}$ \\
\hline Birth weight $>90$ th percentile & $173(12.1)$ & $256(7.4)$ & $<0.01$ \\
\hline Mode of delivery in 2 classes & & & $<0.01$ \\
\hline Vaginal delivery & $924(64.0)$ & $2834(81.8)$ & \\
\hline Cesarean delivery & $520(36.0)$ & $630(18.2)$ & \\
\hline Mode of delivery in 3 classes & & & $<0.01$ \\
\hline Vaginal delivery & $924(64.0)$ & $2834(82.0)$ & \\
\hline Cesarean before labour & $209(14.5)$ & $297(8.5)$ & \\
\hline Cesarean during labour & $311(21.5)$ & $333(9.5)$ & \\
\hline Maternity unit status & & & $<0.01$ \\
\hline Public university & $309(42.2)$ & $1040(30.0)$ & \\
\hline Other public & $626(43.4)$ & $1547(44.7)$ & \\
\hline Private & $209(14.5)$ & $877(25.3)$ & \\
\hline
\end{tabular}

Note: GW = gestational week, $\mathrm{SD}$ = standard deviation .

*Unless stated otherwise.

†Pre-existing chronic medical condition, defined by a binary variable for the presence of at least 1 of the following: hypertension; diabetes; diseases of the circulatory, respiratory or digestive system; hematologic, mental, liver or autoimmune diseases; venous thromboembolism; epilepsy; nephropathy; multiple sclerosis; neoplasia; HIV infection; or active hepatitis B or C.

propensity scores for multiple treatments and "ggplot2" package to create graphics. ${ }^{25}$

\section{Ethics approval}

The study was approved by the National Data Protection Authority (Commission Nationale de l'Informatique et des Libertés [CNIL] authorization no. 912210, Mar. 14, 2012).

\section{Results}

\section{Study population}

Of the women who participated in this study, 1444 were cases and 3464 were controls (Figure 1). Among women with severe acute maternal morbidity, the main underlying causal condition was severe obstetric hemorrhage, observed in 1231 (85.3\%) women (Appendix 5, available at www.cmaj.ca/lookup/suppl/ doi:10.1503/cmaj.181067/-/DC1). Most severe obstetric hemorrhages resulted from uterus atonia (57.9\%). Compared with the control group, women in the case group were significantly more frequently native of sub-Saharan Africa, older, nulliparous or parous with at least 1 previous cesarean, and living without a partner; they also had a higher body mass index and, more often, pre-existing maternal conditions; more previous obstetric hemorrhages; in vitro fertilization; multiple pregnancies; anemia; gestational hypertensive disorders; breech presentations; preterm deliveries; no prophylactic oxytocin after birth; and babies who were larger for gestational age (Table 1). 
The proportion of cesarean delivery was significantly higher among cases than controls (36.0\% v. $18.2 \%$ ). Cesarean deliveries were more frequent among cases both either before labour (14.5\% v. $8.5 \%$ ) or during labour (21.5\% v. $9.5 \%)$.

\section{Propensity score-matched analysis}

Of the 1150 women who had cesarean deliveries, 917 (79.7\%) could be matched with 917 (24.4\%) of the 3758 women who delivered vaginally. In the matched sample, cesarean delivery was associated with an increased risk for severe acute maternal morbidity (adjusted OR 1.8, 95\% Cl 1.5-2.2).

In each maternal age stratum, matched groups were well balanced (Appendix 2B). Compared with vaginal delivery, cesarean delivery was associated with a significantly higher risk for severe acute maternal morbidity in women aged 25 years and older (2529 yr: adjusted OR 1.5, 95\% Cl 1.1-2.2; 30-34 yr: adjusted OR 1.6, $95 \% \mathrm{Cl} 1.2-2.3$; $\geq 35 \mathrm{yr}$ : adjusted OR 2.9, 95\% Cl 1.9-4.4) (Figure 2).

When distinguishing the 3 modes of delivery, the risk for severe acute maternal morbidity was significantly higher with cesarean deliveries during labour at all maternal ages (<25yr: adjusted OR $1.6,95 \% \mathrm{Cl}$ 1.0-2.9; 25-29 yr: adjusted OR 2.0, 95\% Cl 1.3-3.1; 30-34 yr: adjusted OR 2.5, 95\% Cl 1.6-3.9; $\geq 35$ yr: adjusted OR 4.1, 95\% Cl 2.4-6.9). By contrast, the risk for severe acute maternal morbidity with cesarean deliveries before labour was significantly higher only for women aged 35 years and older (adjusted OR 5.1, 95\% Cl 2.3-11.0) (Figure 3).

The sensitivity analysis with inverse propensity score weighting provided similar results (Appendix 3B).

\section{Sensitivity analyses}

Analysis by multivariable logistic regression models in the whole population provided broadly similar results to propensity score estimates (Figures 2 and 3 ).

Analysis by categories of underlying causal condition showed that cesarean delivery was associated with an increased risk of severe obstetric hemorrhage and also with an increased risk of severe acute maternal morbidity from other underlying causal conditions combined (Appendix 6, available at www.cmaj.ca/lookup/suppl/doi:10.1503/cmaj.181067/-/ DC1). Analyses with nonimputed data found similar results (data not shown).

Analysis of the planned mode of delivery showed an increased risk of severe acute maternal morbidity associated with planned cesarean, as compared with planned vaginal delivery, only in women aged 35 years and older (Appendix 7, available at www.cmaj.ca/lookup/suppl/doi:10.1503/cmaj $.181067 /-/ D C 1)$.

\section{Interpretation}

In a prospective population-based analysis focused especially on controlling confounding by indication, cesarean delivery was independently associated with a significantly higher risk of intraor postpartum severe acute maternal morbidity than vaginal delivery. The strength of this association was additionally modified by maternal age. The excess risk was particularly marked for

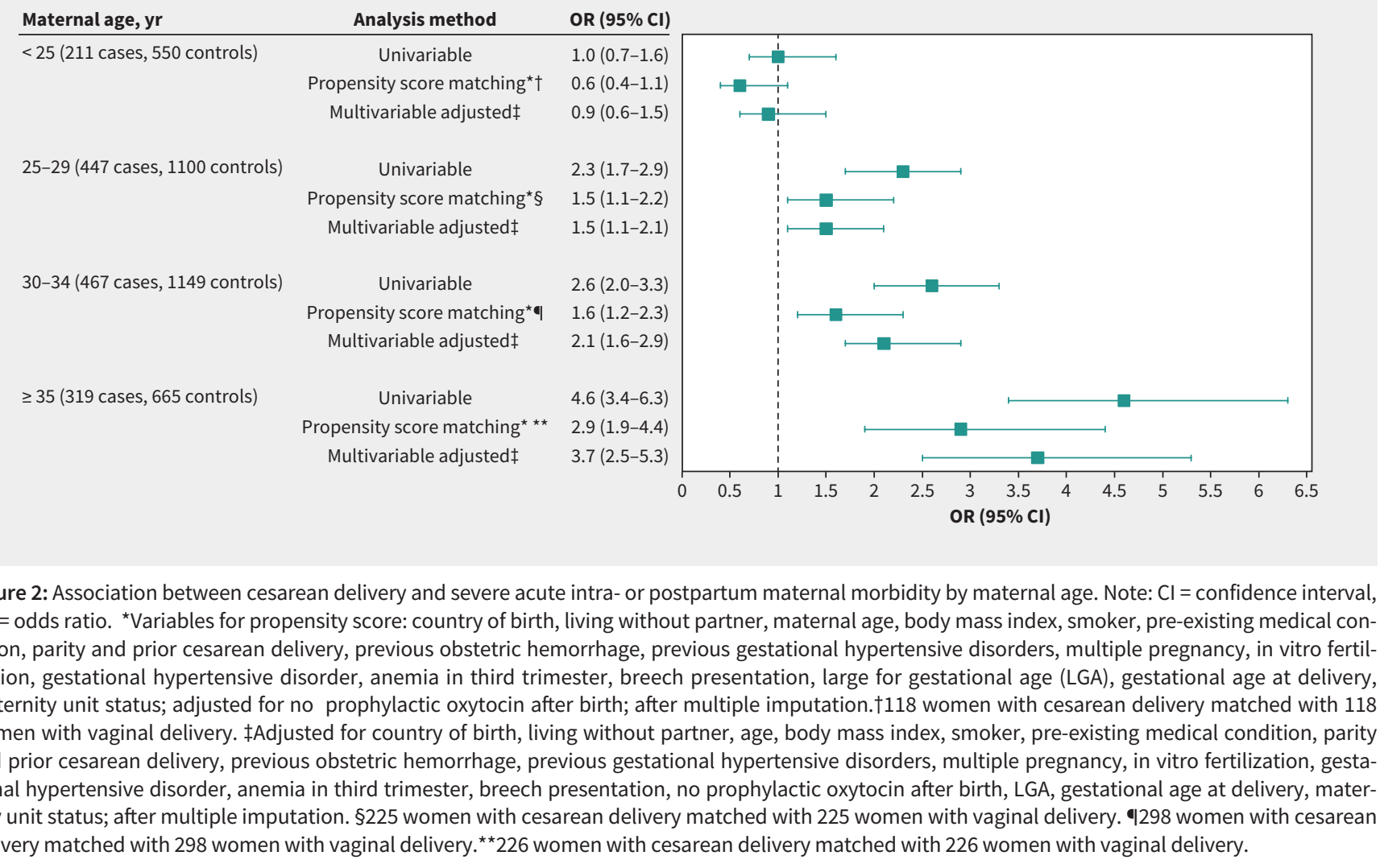


women older than 35 years and existed for cesarean deliveries both before and during labour in this subgroup. The results were consistent for the different statistical methods used to take confounding factors and confounding by indication into account.

Our findings enhance the bulk of evidence that shows that although cesarean delivery is usually a safe intervention, it remains an invasive surgical procedure with intrinsic adverse effects for women. This should be taken into account in the benefit-risk balance when choosing the mode of delivery, together with the reported increased risk of adverse outcomes in babies born by cesarean delivery at short and long term. ${ }^{26-28}$ Indeed, although the literature on this issue is contradictory, some recent studies have reported a higher risk of severe acute maternal morbidity in women who delivered by cesarean delivery, ${ }^{17,18}$ but several methodological limitations raised questions about their results: the retrospective design limits the quality and availability of data, and in consequence potentially leads to inaccurate characterization of women with severe acute maternal morbidity and of the

A

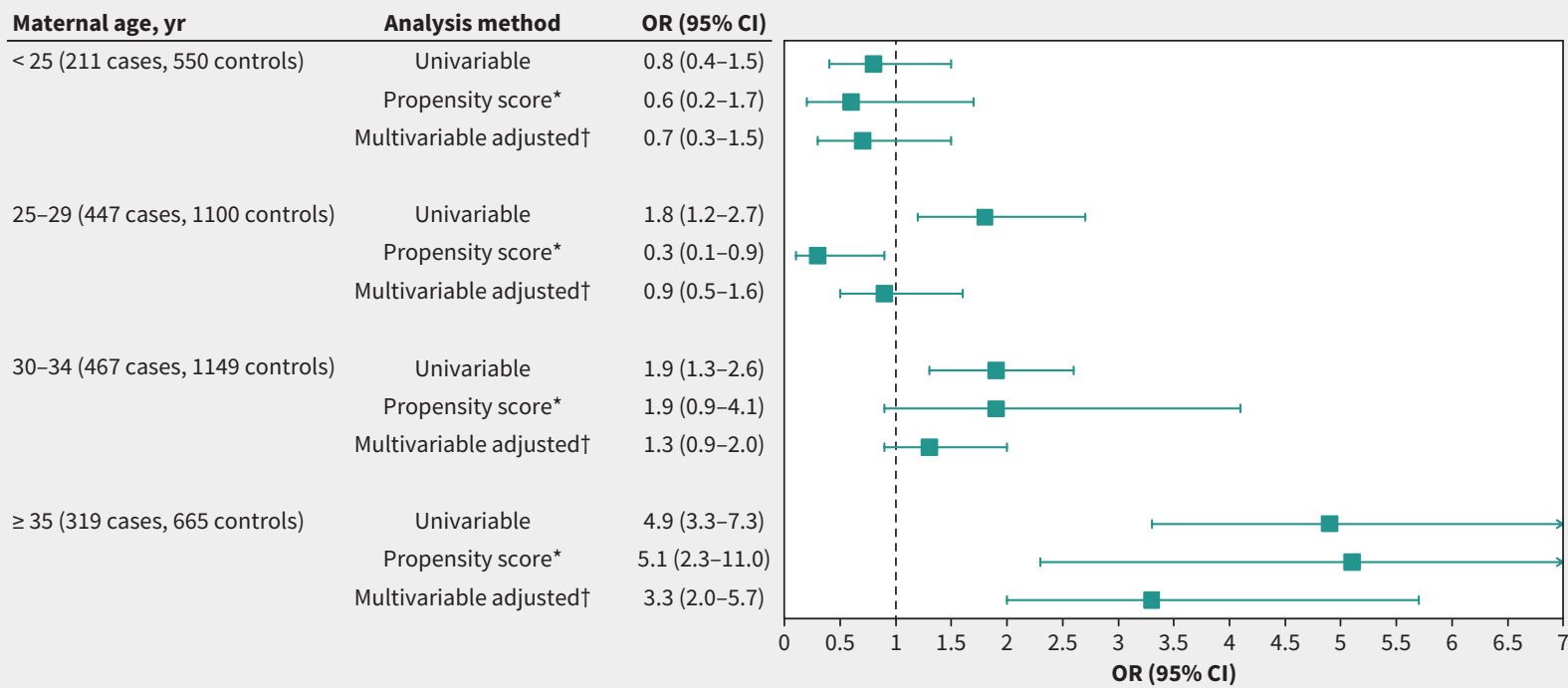

B

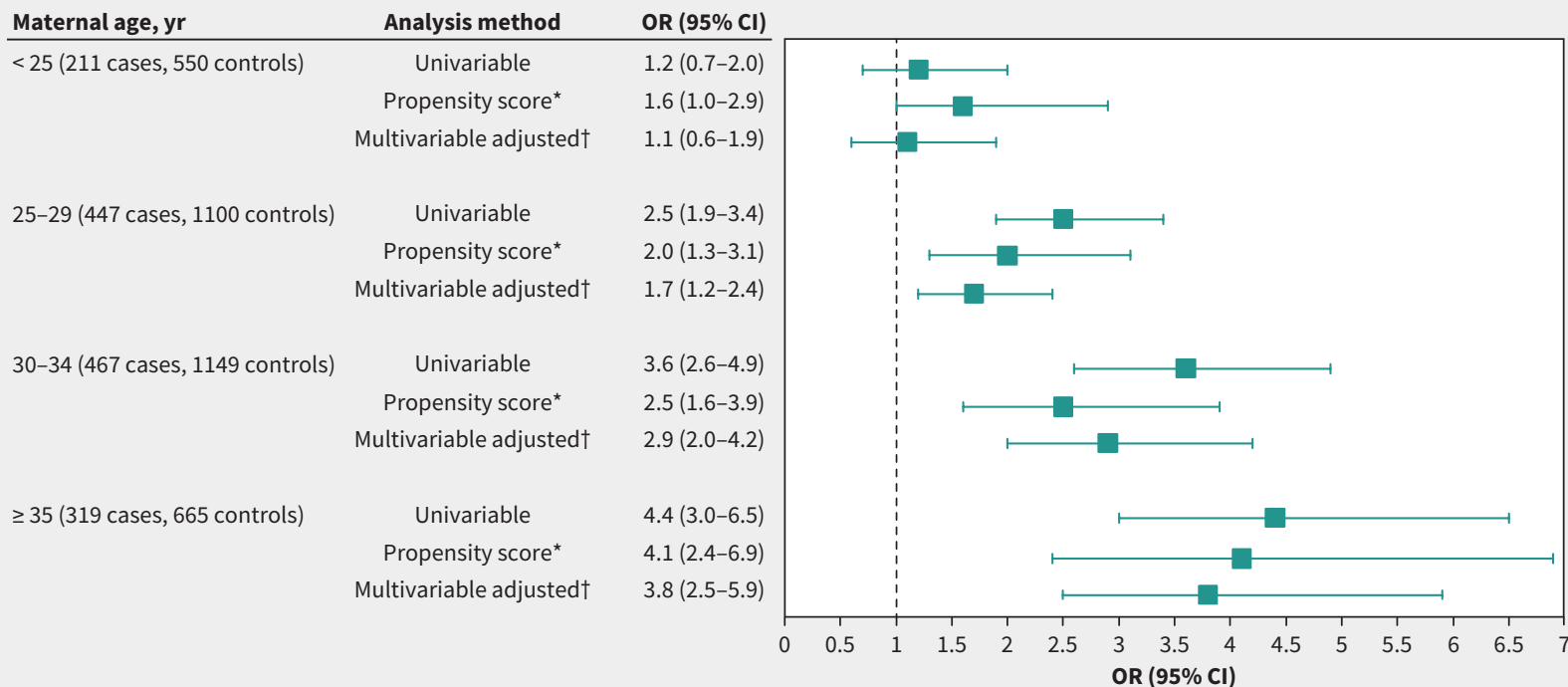

Figure 3: Association between cesarean delivery (A) before and (B) during labour, and severe acute intra- or postpartum maternal morbidity by maternal age. Note: $\mathrm{Cl}$ = confidence interval, $\mathrm{OR}=$ odds ratio. *Variables for propensity score: country of birth, living without partner, age, body mass index (BMI), current smoker, pre-existing medical condition, parity and prior cesarean delivery, previous obstetric hemorrhage, previous gestational hypertensive disorders, multiple pregnancy, in vitro fertilization, gestational hypertensive disorder, anemia in third trimester, breech presentation, large for gestational age (LGA), gestational age at delivery and maternity unit status; adjusted for no prophylactic oxytocin after birth; after multiple imputation. †Adjusted for country of birth, living without partner, age, BMI, current smoker, pre-existing medical condition, parity and prior cesarean delivery, previous obstetric hemorrhage, previous gestational hypertensive disorders, multiple pregnancy, in vitro fertilization, gestational hypertensive disorder, anemia in third trimester, breech presentation, no prophylactic oxytocin after birth, LGA, gestational age at delivery and maternity unit status; after multiple imputation. 
timing of the cesarean delivery, and to a limited number of available covariables. Additional limitations have included insufficient consideration of confounding by indication performed through the selection of a low-risk population; definition of severe acute maternal morbidity limited to selected components of severe maternal morbidity, which does not allow for a comprehensive study of severe maternal complications; and use of data from hospital databases that do not allow for accurate and valid characterization of women or of morbidity events.

Using prospective data and a strong methodology to avoid indication bias, we found that cesarean delivery is independently associated with a significantly higher risk of severe acute maternal morbidity than vaginal delivery. Our main analysis was deliberately performed according to the actual mode of delivery and not according to the planned mode of delivery, for different reasons. First, from an explanatory or causal perspective, to isolate the intrinsic risk of severe maternal morbidity associated with cesarean delivery, it is more relevant to conduct an analysis according to the received mode of delivery, which is the actual exposure. Conversely, when the objective is to guide clinical practice in a specific context where there is a debate on what strategy for delivery should be chosen first, an analysis by intended mode of delivery will answer the question. In addition, an analysis by intended mode of delivery in the overall population of parturients will be very much affected by the initial selection and proportion of women with planned cesarean delivery, and then by the selection and proportion of cesarean deliveries during labour (this last route of delivery being the most at risk), both highly dependent on obstetric practices. We nevertheless also conducted a sensitivity analysis with the intended mode of delivery; its results were consistent with our main analysis, showing an increased risk of severe maternal morbidity associated with planned cesarean delivery in women aged 35 years and older, a result similar to that observed for cesarean delivery performed before labour. This was an expected finding, as most planned cesarean deliveries are performed before labour.

Another important result is that the risk of severe acute maternal morbidity associated with cesarean delivery is modified by maternal age. In higher-income countries, maternal age at delivery has increased during recent decades; women older than 35 years account for a substantial proportion of pregnancies $-17.0 \%$ in the United States, $21 \%$ in France and in England and $21.7 \%$ in Canada in 2016. ${ }^{29-32}$ Concomitantly, the rate of cesarean delivery has risen, especially in women older than 35 years, with rates around $30 \%$ reported in this older age group. ${ }^{33,34}$ Older maternal age has been associated with more pre-existing conditions, more obstetric complications, resulting in more maternal morbidity, and with an increased risk of progression from severe maternal morbidity to death. ${ }^{35-38}$ We adjusted for known pre-existing conditions in our analysis. It is nonetheless possible that preclinical alterations of organ function owing to aging limit the potential of some women to recover after stress such as surgery and explain the effect of age on these maternal complications of cesarean delivery. Such changes have been reported in nonobstetric contexts, including resuscitation after sepsis or hemorrhagic shock, and may reflect a lack of physiologic robustness to respond to pathology with older age..$^{39,40}$ In the obstetrics setting, various studies have reported an increased risk of postpartum hemorrhage with advanced maternal age; $38,41-45$ pathophysiological data also support this mechanism, including changes in myometrial contractility with maternal age, which may cause severe obstetric hemorrhage. ${ }^{46,47}$

This study has several strengths. It was based on a population-based prospective cohort study, which enables us to consider the diversity of practices and of women's characteristics. Both the use of a standardized and comprehensive definition of severe acute maternal morbidity based on a national consensus and the prospective identification of women meeting this definition limited the risk of selection bias for cases. The method we used to select the women in the control group as a representative sample of the source cohort makes selection bias unlikely for them as well. The study design allowed for the collection of covariables and potential confounding factors absent in studies conducted from hospital databases. ${ }^{17,18}$ The accuracy of the prospective data collection of the EPIMOMS study makes it possible to distinguish with certainty cesarean before labour and cesarean during labour, which is known to offer more at risk of intra- or postpartum severe acute maternal morbidity. ${ }^{17}$ Finally, through diverse statistical approaches, we also took into account the confounding by indication inherent in this type of observational study. We performed a propensity score analysis and made extensive adjustments for confounding factors to minimize the likelihood of incorrectly attributing any risk of intra- or postpartum severe acute maternal morbidity to cesarean delivery.

\section{Limitations}

The main limitation of this study is its observational nature and therefore, despite the large number of covariables available, we cannot exclude the presence of a possible unmeasured residual confounding, in particular for intrapartum cesarean. Furthermore, for maternal conditions that were not excluded, adjustment for complications or diseases that existed before delivery as binary variables, or inclusion of these variables in the propensity score, may not completely control for confounding, if the severity within pre-existing conditions or categories of pregnancy complications was heterogeneous, which could also lead to possible, although marginal, residual confounding. However, such a residual confounding seems unlikely to explain the strength of the association found here.

This study was conducted in France, which may limit the external validity of the results for other countries. However, the rate and practices of cesarean delivery in France, ${ }_{2}$ as well as its overall rates of maternal mortality and severe morbidity, are similar to those in other high-resource countries. ${ }^{1,33,34}$ The predominance of severe obstetric hemorrhage as the main underlying causal condition of intra- or postpartum severe acute maternal morbidity, although standard in all similar studies, ${ }^{17,18}$ is even stronger in the current study because of the selection of postpartum morbidity cases designed to limit confounding by indication. However, we also found that the risk of severe acute maternal morbidity from other causes combined was increased with 
cesarean delivery, which shows that the cesarean deliveryrelated maternal risks are not limited to bleeding.

Finally, the rarity of each of the other underlying causal conditions of severe acute maternal morbidity limited specific analyses of their association with cesarean.

\section{Conclusion}

We found that cesarean delivery was associated with a higher risk of severe acute maternal morbidity than vaginal delivery, particularly in women aged 35 years and older. This result has implications for clinical practice and will be useful in deciding the mode of delivery. Our finding raises questions about the practices of some obstetricians who may consider cesarean deliveries to be indicated by advanced maternal age, with the idea that there will probably be no further pregnancies. This practice should be modified to avoid unnecessarily exposing women older than 35 years to the excess risk of severe acute maternal morbidity. In addition, when a cesarean delivery is necessary, our finding should encourage caregivers to prepare more proactively for the potential severe acute maternal morbidity that may result.

\section{References}

1. The European Perinatal Health Report: health and care of pregnant women and babies in Europe in 2010. Euro-Peristat; 2013. Available: www.europeristat .com/Reports/European-Perinatal-Health-Report-2010.Html (accessed 2018 Aug. 10).

2. Scott JR. Cesarean delivery on request: Where do we go from here? Obstet Gynecol 2006;107:1222-3.

3. Meikle SF, Steiner CA, Zhang J, et al. A national estimate of the elective primary cesarean delivery rate. Obstet Gynecol 2005;105:751-6.

4. Parrish KM, Holt VL, Easterling TR, et al. Effect of changes in maternal age, parity, and birth weight distribution on primary cesarean delivery rates. JAMA 1994;271:443-7.

5. Hannah ME. Planned elective cesarean section: a reasonable choice for some women? CMAJ 2004;170:813-4.

6. American College of Obstetricians and Gynecologists. ACOG Committee Opinion. Surgery and patient choice: the ethics of decision making. Obstet Gynecol 2003;102:1101-6.

7. Declercq E, Menacker F, MacDorman M. Rise in "no indicated risk" primary caesareans in the United States, 1991-2001: cross sectional analysis. BMJ 2005;330:71-2.

8. Guise J-M, Eden K, Emeis C, et al. Vaginal birth after cesarean: new insights. Evid Rep Technol Assess (Full Rep) 2010;(191):1-397.

9. Rosenberg T, Pariente G, Sergienko R, et al. Critical analysis of risk factors and outcome of placenta previa. Arch Gynecol Obstet 2011;284:47-51.

10. Vaginal birth after cesarean: new insights. AHRQ Publication No. 10-E003. Rockville (MD): Agency for Healthcare Research and Quality; 2010.

11. Zwart JJ, Richters JM, Ory F, et al. Uterine rupture in The Netherlands: a nationwide population-based cohort study. BJOG 2009;116:1069-78, discussion 1078-80.

12. Al-Zirqi I, Stray-Pedersen B, Forsén L, et al. Uterine rupture after previous caesarean section. BJOG 2010;117:809-20.

13. Fitzpatrick KE, Kurinczuk JJ, Alfirevic Z, et al. Uterine rupture by intended mode of delivery in the UK: a national case-control study. PLoS Med 2012;9:e1001184.

14. Hall MH, Bewley S. Maternal mortality and mode of delivery. Lancet 1999;354:776.

15. Deneux-Tharaux C, Carmona E, Bouvier-Colle M-H, et al. Postpartum maternal mortality and cesarean delivery. Obstet Gynecol 2006;108:541-8.
16. Villar J, Carroli G, Zavaleta N, et al.; World Health Organization 2005 Global Survey on Maternal and Perinatal Health Research Group. Maternal and neonatal individual risks and benefits associated with caesarean delivery: multicentre prospective study. BMJ 2007;335:1025.

17. Liu S, Liston RM, Joseph KS, et al.; Maternal Health Study Group of the Canadian Perinatal Surveillance System. Maternal mortality and severe morbidity associated with low-risk planned cesarean delivery versus planned vaginal delivery at term. CMAJ 2007;176:455-60.

18. Curtin SC, Gregory KD, Korst LM, et al. Maternal morbidity for vaginal and cesarean deliveries, according to previous cesarean history: new data from the birth certificate, 2013. Natl Vital Stat Rep 2015;64:1-13.

19. National Collaborating Centre for Women's and Children's Health (UK). Caesarean section. London (UK): RCOG Press; 2011. Available: www.ncbi.nlm.nih.gov/books/ NBK115309/ (accessed 2015 Jan. 4).

20. NIH State-of-the-Science Conference Statement on cesarean delivery on maternal request. NIH Consens State Sci Statements 2006;23:1-29.

21. Blondel B, Lelong N, Kermarrec M, et al.; National Coordination Group of the National Perinatal Surveys. Trends in perinatal health in France from 1995 to 2010. Results from the French National Perinatal Surveys. J Gynecol Obstet Biol Reprod (Paris) 2012;41:e1-15.

22. McCaffrey DF, Griffin BA, Almirall D, et al. A tutorial on propensity score estimation for multiple treatments using generalized boosted models. Stat Med 2013;32:3388-414.

23. Garrido MM, Kelley AS, Paris J, et al. Methods for constructing and assessing propensity scores. Health Serv Res 2014;49:1701-20.

24. Leuven E, Sianesi B. PSMATCH2: stata module to perform full Mahalanobis and propensity score matching, common support graphing, and covariate imbalance testing. Statistical Software Components S432001. Chestnut Hill (MA): Department of Economics, Boston College; revised 2018 Feb. 1. Available: https://ideas.repec. org/c/boc/bocode/s432001.html (accessed 2018 Aug. 10).

25. Ridgeway G, McCaffrey D, Morral A, et al. Toolkit for weighting and analysis of non-equivalent groups: a tutorial for the twang package. 2017 July 1. Available: https://cran.r-project.org/web/packages/twang/vignettes/twang.pdf (accessed 2018 Aug. 10).

26. Hansen AK, Wisborg K, Uldbjerg N, et al. Risk of respiratory morbidity in term infants delivered by elective caesarean section: cohort study. BMJ 2008; 336:85-7.

27. Keag OE, Norman JE, Stock SJ. Long-term risks and benefits associated with cesarean delivery for mother, baby, and subsequent pregnancies: systematic review and meta-analysis. PLoS Med 2018;15:e1002494.

28. Sandall J, Tribe RM, Avery L, et al. Short-term and long-term effects of caesarean section on the health of women and children. Lancet 2018;392:1349-57.

29. Martin JA, Hamilton BE, Osterman MJK, et al. Births: final data for 2016. In: National Vital Statistics Reports. Vol 67, no 1. Hyattsville (MD): US Department of Health and Human Services, National Center for Health Statistics; 2018 Jan. 31.

30. NHS maternity statistics - England, 2014-15. Leeds (UK): NHS Digital; 2015 Nov. 25.

31. Kenny LC, Lavender T, McNamee R, et al. Advanced maternal age and adverse pregnancy outcome: evidence from a large contemporary cohort. PLoS One 2013;8:e56583.

32. Tableau 13-10-0416-01: Naissances vivantes, selon l'âge de la mère. Ottawa: Statistics Canada; modified 2019 Mar. 4. Available: https://www150.statcan. gc.ca/t1/tbl1/fr/tv.action?pid=1310041601 (accessed 2018 Aug. 8).

33. Lisonkova S, Potts J, Muraca GM, et al. Maternal age and severe maternal morbidity: a population-based retrospective cohort study. PLoS Med 2017;14: e1002307.

34. European Health for All databases (HFA-DB). Copenhagen: Division of Information, Evidence, Research and Innovation, WHO Regional Office for Europe; updated 2018 June 15. Available: http://data.euro.who.int/hfadb/ (accessed 2018 Aug. 8).

35. Kayem G, Kurinczuk J, Lewis G, et al. Risk factors for progression from severe maternal morbidity to death: a national cohort study. PLoS One 2011;6:e29077. 
36. Laopaiboon M, Lumbiganon P, Intarut N, et al.; WHO Multicountry Survey on Maternal Newborn Health Research Network. Advanced maternal age and pregnancy outcomes: a multicountry assessment. BJOG 2014;121(Suppl 1):49-56.

37. Gilbert WM, Nesbitt TS, Danielsen B. Childbearing beyond age 40: pregnancy outcome in 24,032 cases. Obstet Gynecol 1999;93:9-14.

38. Luke B, Brown MB. Elevated risks of pregnancy complications and adverse outcomes with increasing maternal age. Hum Reprod 2007;22:1264-72.

39. Martin GS, Mannino DM, Moss M. The effect of age on the development and outcome of adult sepsis. Crit Care Med 2006;34:15-21.

40. Vanzant EL, Hilton RE, Lopez CM, et al.; Inflammation and Host Response to Injury Investigators. Advanced age is associated with worsened outcomes and a unique genomic response in severely injured patients with hemorrhagic shock. Crit Care 2015;19:77.

41. Herstad L, Klungsøyr K, Skjærven R, et al. Elective cesarean section or not? Maternal age and risk of adverse outcomes at term: a population-based registry study of lowrisk primiparous women. BMC Pregnancy Childbirth 2016;16:230.
42. Osmundson SS, Gould JB, Butwick AJ, et al. Labor outcome at extremely advanced maternal age. Am J Obstet Gynecol 2016;214:362.e1-7.

43. Yogev Y, Melamed N, Bardin R, et al. Pregnancy outcome at extremely advanced maternal age. Am J Obstet Gynecol 2010;203:558.e1-7.

44. Ananth CV, Wilcox AJ, Savitz DA, et al. Effect of maternal age and parity on the risk of uteroplacental bleeding disorders in pregnancy. Obstet Gynecol 1996;88: 511-6.

45. Ohkuchi A, Onagawa $\mathrm{T}$, Usui R, et al. Effect of maternal age on blood loss during parturition: a retrospective multivariate analysis of 10,053 cases. J Perinat Med 2003;31:209-15.

46. Arrowsmith S, Robinson H, Noble K, et al. What do we know about what happens to myometrial function as women age? J Muscle Res Cell Motil 2012;33: 209-17.

47. Elmes M, Szyszka A, Pauliat C, et al. Maternal age effects on myometrial expression of contractile proteins, uterine gene expression, and contractile activity during labor in the rat. Physiol Rep 2015;3. pii: e12305. doi: 10.14814/phy2.12305.

\section{Competing interests: None declared.}

This article has been peer reviewed.

Affiliations: INSERM U1153 (Korb, Goffinet, Seco, Deneux-Tharaux), Obstetrical, Perinatal and Pediatric Epidemiology Research Team (EPOPé), Research Center for Epidemiology and Biostatistics Sorbonne Paris Cité (CRESS), Paris Descartes University; Department of Obstetrics and Gynecology (Korb), Robert Debré Hospital, l'Assistance publique - hôpitaux de Paris (APHP); Port-Royal Maternity Unit (Goffinet), Cochin Hospital, APHP; Clinical Research Unit of Paris Descartes Necker Cochin (Seco), APHP; Biostatistics and Clinical Epidemiology (Chevret), ECSTRA Team, INSERM, Paris Diderot Sorbonne University, Paris, France

Contributors: Catherine Deneux-Tharaux, François Goffinet and Diane Korb had full access to all the data in the study and take responsibility for the integrity of the data and the accuracy of the data analysis. Catherine Deneux-Tharaux, François Goffinet, Sylvie Chevret and Diane Korb conceptualized the study and wrote the manuscript. Diane Korb and Aurélien Seco per- formed the statistical analysis. Catherine Deneux-Tharaux obtained funding and supervised the study. All of the authors contributed to the interpretation of the data, revised the manuscript critically for important intellectual content, gave final approval of the version to be published and agreed to be accountable for all aspects of the work.

Funding: Supported by a grant from the National Research Agency and the île-deFrance Regional Health Agency.

Acknowledgements: The authors thank the coordinators of the participating regional perinatal networks: Alsace, Aurore, Auvergne, BasseNormandie, MYPA, NEF, Paris Nord, 92 Nord, Lorraine; Chloé Barasinski, Sophie Bedel, Aline Clin D'Amour, Laurent Gaucher, Isabelle Le Creff, Blandine Masson Carole Ramousset, Mathias Rossignol, Zelda Stewart, Dalila Talaourar Yacine Toure, Nicole Wirth for their contribution to the implementation of the EPIMOMS study in their region; the obstetricians, midwives and anesthetists who contributed to case identification and documentation in their hospital; and the research assistants who collected the data.
EPIMOMS Study Group: Bruno Langer, Naitre en Alsace Perinatal Network; Corinne Dupont and René-Charles Rudigoz, Rhône-Alpes Aurore Perinatal Network; Françoise Vendittelli, Auvergne Perinatal Network; Gaël Beucher, Basse-Normandie Perinatal Network; Patrick Rozenberg, MYPA Perinatal Network, lle de France region; Lionel Carbillon, Naitre dans l'Est Francilien Network, île-deFrance region; Elie Azria and Nathalie Baunot, Paris Nord Perinatal Network; Catherine Crenn-Hebert and Gilles Kayem, 92 Nord Perinatal Network, île-de-France region; Jeanne Fresson, Lorraine Perinatal Network; Alexandre Mignon, Société Française d'Anesthésie Réanimation; Sandrine Touzet, Santé Publique pole, Hospices Civils de Lyon; Marie-Pierre Bonnet, Marie-Hélène BouvierColle, Anne Chantry, Coralie Chiesa-Dubruille, Catherine Deneux-Tharaux and Aurélien Seco, Inserm EPOPé research team.

Accepted: Mar. 1, 2019

Correspondence to: Diane Korb, diane.korb@inserm.fr 